\title{
La formación de postgrado y la investigación en Educación Ambiental en España: E1 Programa Interuniversitario de Doctorado en Educación Ambiental
}

Lucía Iglesias da Cunha ${ }^{1}$

Resumen: La Educación Ambiental es un foco de interés científico pujante. Tanto es así que atrae los intereses de diversas áreas de conocimiento como puedan ser las ciencias de la educación, las ciencias ambientales y otras como la psicología, la sociología, la política, etc. El debate sobre la conveniencia o no de que constituya una materia de formación curricular se decanta la mayoría de las veces por la opción de la transversalidad, pero lo cierto es que en aquellos casos en que se da formación específica en Educación Ambiental, ésta se refuerza. Es el caso del Programa Interuniversitario de Doctorado en Educación Ambiental que durante dos décadas pudo ofrecer formación específica de los campos disciplinares que construyen conocimiento científico y profesional relacionados con la Educación Ambiental.

Palabras-clave: Educación Ambiental, formación universitaria en España, investigación en Educación Ambiental.

Graduate Training in Environmental Education and Research in Spain. The Inter-University PhD Program in Environmental Education.

Abstract: Environmental education is a strong focus of scientific interest. Therefore show interest knowledge areas of science education, environmental sciences, psychology, sociology, politics, etc. The debate about the convenience or not an environmental education training curriculum constitutes a training curriculum is decanted mostly by choice of the transversality. But the truth is that in those cases where there is specific training in environmental education, it is strengthened. This is the

1 La Profesora Doctora Lucía Iglesias da Cunha está integrada en el Equipo de Investigación "Pedagogía Social y Educación Ambiental" (SEPA) de la Universidad de Santiago de Compostela, que forma parte de la "Rede de Investigación en Educación e Formación para a Cidadanía e a Sociedade do Coñecemento" (RINEF-CISOC), financiada por la Xunta de Galicia-España con fondos FEDER de la Unión Europea. Santiago de Compostela, Espanha, lucia.dacunha@usc.es 
case of Spanish Interuniversity Doctoral Program in Environmental Education for two decades could offer specific training of disciplinary fields that build scientific and professional knowledge related to environmental education.

Keywords: Environmental Education, university education in Spain, Environmental Education research

\section{EDUCACIÓN AMBIENTAL EN LOS ESTUDIOS UNIVERSITARIOS DE POSGRADO Y DOCTORADO EN ESPAÑA}

$\mathrm{El}$ aumento del volumen de estudiantes universitarios que ha vivido España desde los años setenta, y mayormente desde los años ochenta, tiene un efecto positivo sobre la tasa de matrícula en enseñanza superior en edades entre 18-24 años. En el curso 2009-2010 se alcanzó una tasa del 24,5\%, cifra que supone un crecimiento de un $0,7 \%$ con respecto al curso anterior ${ }^{2}$. Las razones son, por un lado, el incremento general de la matrícula en estudios universitarios $(2 \%)$, y por otro, una reducción del 3,4\% de la población entre 18 y 24 años (Ministerio de Educación: 2010: 8). El incremento del volumen de estudiantes universitarios vivido a partir de finales de los años setenta, y mayormente en la década de los ochenta, puso las bases para que actualmente el $29 \%$ de la población española adulta entre 25 y 64 años tenga estudios superiores. Comparativamente la Unión Europea (UE-19) alcanza un 25\% y los países de la OCDE un 28\% (Ministerio de Educación, 2010: 98).

${ }^{2}$ En España, existen 50 Universidades públicas que atienden al 88,9\% de los estudiantes de nivel superior. Las Universidades privadas que matriculan al 11,1\% son 24 (de las que 7 pertenecen a la Iglesia Católica) y hay otros centros emergentes (datos del Instituto Nacional de Estadística, curso 2009-2010). La matrícula en cursos de Máster en universidades públicas asciende al $86,7 \%$ y alcanza un $94,9 \%$ cuando se trata de estudios de doctorado (Ministerio de Educación, 2010: 4).

${ }^{3}$ Las universidades españolas han tenido que modificar las estructuras de sus cursos de doctorado por las de cursos Máster con la entrada en vigor del RD 56/2005 y posteriormente del RD 1393/2007. Así, los programas formativos de dos años se transforman en cursos de un solo año, y los «Diplomas de Estudios Avanzados» desaparecen como acreditación de la suficiencia investigadora. Alcanzar el título de Máster de investigación es ahora la condición para inscribirse en un Programa de Doctorado con un proyecto de Tesis doctoral. De este modo el cómputo de los estudiantes se diferencia entre los que están cursando un Máster (que equivaldría a un segundo ciclo) y los que están en periodo de investigación del doctorado equivalente al tercer ciclo (Ministerio de Educación: 2010: 8). 
Esta dinámica de incremento del nivel de formación de la población española generó un impulso similar en la cantidad de personas que iniciaban estudios de doctorado, que lograban acreditar Suficiencia Investigadora, Diploma de Estudios Avanzados o graduarse como Máster según la normativa vigente en cada caso (Ley de Reforma Universitaria LRU de 1983 y Real Decreto 778/98 que regulaba los estudios de Tercer Ciclo, la expedición del título de doctor y otros estudios de postgrado; Ley de Ordenación Universitaria LOU de 2005 y Real Decreto 56/2005 sobre los estudios oficiales de postgrado y posteriormente Real Decreto 1393/2007 sobre la ordenación de las enseñanzas universitarias de Grado, Máster y Doctorado ${ }^{3}$. Si las cifras de las universidades españolas apuntan a un ascenso sin precedentes, podemos indicar que se reproduce la misma pauta en lo que respecta a los estudios de Ciencias de la Educación.

De todos modos, debe advertirse que la disciplina de Educación Ambiental tiene un tratamiento desigual en las Universidades españolas. Como materia a impartir en los estudios de Grado su presencia es mayor en las titulaciones ligadas a las Ciencias de la Educación, pero no en todas y no en todas las universidades ${ }^{4}$. En tanto que, su presencia en los estudios de postgrado permite observar la disparidad de ópticas científicas desde las que se trabaja y se profundiza en la investigación en Educación Ambiental. En España las temáticas docentes e investigadoras están enmarcadas en un "catálogo de áreas de conocimiento" que desarrollan proyectos docentes y de investigación cada vez más especializados. A pesar de que este catálogo está siempre sujeto a controversias y debates, es la base sobre la que se organiza el cuadro de personal docente e investigador, así como la oferta de cursos y titulaciones.

\section{EL PROGRAMA DE DOCTORADO INTERUNIVERSITARIO EN EDUCACIÓN AMBIENTAL}

El Programa Interuniversitaro de Educación Ambiental supuso una innovación importante en la oferta formativa de cursos dirigidos a formar investigadoras e investigadores, así como a potenciar la realización de Tesis doctorales sobre Educación Ambiental. Las nueve universidades participantes ${ }^{5}$

\footnotetext{
4 Estudios de Magisterio, Pedagogía, Educación Social serán los que mayormente han introducido la disciplina de Educación Ambiental con variantes en la denominación concreta de la misma.

5 En el Programa Interuniversitario de Educación Ambiental participan las siguientes universidades: Autónoma de Barcelona (UAB), Autónoma de Madrid (UAM), Gerona (UdG), Granada (UGR), Islas Baleares (UIB), La Laguna (ULL), Santiago de Compostela (USC), Sevilla (US) y Valencia (UV).
} 
aportan sus activos en cuanto a experiencia docente y trayectoria científica de su profesorado más implicado en cuestiones educativo-ambientales.

En el curso 1998-99, más de medio centenar de profesoras y profesores de diferentes universidades españolas mantuvieron una reunión en el Centro Nacional de Educación Ambiental (CENEAM) situado en Valsaín-Segovia, para debatir sobre la oportunidad, la necesidad y las posibilidades de organizar un programa de doctorado especializado en Educación Ambiental. De ahí surgió la idea, el cuadro de docentes, la estructura del curso, el nombramiento de coordinadores y todo lo necesario para presentar la documentación en las nueve universidades que participarían. La fuerza de esta propuesta residía en que cada una de las universidades aportaba su profesorado especializado en Educación Ambiental y en la mayoría de casos ya dirigían trabajos de investigación en este campo (Javier Benayas, área de Ecología; José Gutiérrez, área de Métodos de Investigación en Educación; Rosa María Pujol, área de Didáctica de las Ciencias Experimentales; Pablo Meira, área de Teoría e Historia de la Educación; José Antonio Corraliza, área de Psicología Social, etc.).

Es necesario reparar en que hay más personas relevantes en la construcción de la disciplina en España como puedan ser José Antonio Caride Gómez (USC), María Novo Villaverde (UNED), José Antoni Colom Cañellas (UIB), Fernando González Bernáldez (UAM) que fueron los precursores desde las Ciencias de la Educación los tres primeros y desde la Ecología el último, en señalar este campo como uno de los intereses científicos de la producción investigadora española. Hay, desde luego, más personas y alguna que otra universidad más que, dentro de programas de doctorado propios de sus áreas de conocimiento respectivas, imparten y dirigen trabajos relacionados con la Educación Ambiental. Es por ello que el Programa Interuniversitario de Doctorado en Educación Ambiental no capitalizaba toda la formación ni producción científica al respecto.

El Programa definió tres grandes elementos como objetivos formales del mismo:

-Promover la formación de nuevos investigadores/as en el campo de la Educación Ambiental en las universidades implicadas.

-Potenciar la calidad de la investigación en esta temática en los distintos departamentos de dichas universidades.

-Promover la formación en esta especialidad por parte de estudiantes de áreas afines.

El primero, promover la formación de nuevos investigadores/as en el campo, tiene su justificación en el hecho de que la formación en investigación sobre Educación Ambiental figuraba como contenido de múltiples cursos de doctorado de las universidades españolas avalados por personas afines al ambientalismo desde diferentes ópticas: Ecología, Pedagogía, Psicología, Didáctica de las Ciencias, etc. La reunión académica citada anteriormente no hizo más que poner en evidencia el deseo común de hacer confluir a todas estas 
personas (o a su gran mayoría) en un solo curso y poder ofertar un programa de doctorado especializado en Educación Ambiental. De este modo la persona que, finalizados sus estudios de Licenciatura (Grado), tuviese cierta claridad en el enfoque de sus intereses hacia la investigación en Educación Ambiental no se vería obligada a estudiar un "doctorado genérico" en cualquiera de las ramas científicas antes mencionadas, para posteriormente tener el "aprendizaje especializado" cuando se desarrollase su trabajo de Tesis doctoral. Había, pues, la necesidad de ofertar un espacio formativo especializado en la investigación en Educación Ambiental en el que se pudiesen concentrar diversas disciplinas que construyen el conocimiento de este campo científico. La interdisciplinariedad siempre fue una de las características que definieron la investigación en Educación Ambiental, por lo que se consideró necesario que constituyese la caracterización de la formación de nuevas generaciones que iban a investigar en él; por lo tanto, la formación de postgrado en Educación Ambiental precisaba una construcción interdisciplinar de sus aprendizajes iniciales.

El segundo, potenciar la calidad de la investigación en Educación Ambiental, siendo un objetivo perseguible por cualquier oferta formativa, en este caso, tiene un componente de autoanálisis previo. Los trabajos realizados por Javier Benayas y José Gutiérrez (Benayas, 1996; Benayas, Gutiérrez y Hernández, 2003) sobre la producción de Tesis doctorales sobre Educación Ambiental en España revelaban el incremento exponencial iniciado en 1978 con una Tesis sobre Ecología y Educación ${ }^{6}$, que progresivamente se duplicaba aproximadamente- cada cinco años (1-2-7-17-32..). El volumen de trabajos ya permitía realizar análisis sobre las temáticas que se estaban investigando, las metodologías de investigación empleadas, las personas que dirigían los trabajos y los equipos que se estaban conformando.

La calidad también se refiere a asegurar que la producción científica contribuya a aumentar el conocimiento de la Educación Ambiental y no se desvíe de sus principales focos de interés. Es verdad que los trabajos incorporan en sus títulos los términos "educación ambiental" u otros afines que permiten clasificar de inmediato una Tesis doctoral como propia del campo. Sin embargo,

\footnotetext{
${ }^{6}$ Se trata del trabajo de Rafael Salord Barceló dirigido por Alejandro Sanvisens Marfull de la Facultad de Filosofía y Ciencias de la Educación de la Universidad Autónoma de Barcelona.
} 
los debates sobre la investigación en Educación Ambiental alertan sobre la necesidad de exigir un mayor equilibrio en la presencia de aspectos educativos y ambientales en los trabajos de investigación sobre Educación Ambiental. Así, mejorar las condiciones a partir de las que se inician los trabajos de investigación, fundamentalmente relacionadas con los conocimientos que se adquieren y experiencias que se facilitan, es un modo de fomentar la realización de mejores trabajos de investigación en Educación Ambiental.

El tercero, formar en la especialidad a estudiantes afines, se relaciona con el primero y amplía su perspectiva de captar personas interesadas en el amplísimo espectro de titulaciones existentes hoy en día. Las Ciencias de la Educación y las Ciencias Ambientales son las áreas científicas que mayor tradición tienen en el interés por la Educación Ambiental. No es de extrañar, por tanto, que en sus titulaciones de Pedagogía, Magisterio, Ecología o Biología hayan incorporado formación específica sobre Educación Ambiental en algunas universidades españolas ${ }^{7}$. Lo cierto es que esta temática suscita interés en otras ciencias y en otras personas que se interesan por iniciarse en la investigación de este campo. Así, personas con formación en Física, Química, Ingeniería, Geología, Derecho, Humanidades, Psicología, Geografía, etc. pueden obtener una formación especializada y al tiempo que complementan sus saberes, enriquecen los cursos de los que forman parte, porque aportan sus conocimientos, sus experiencias y sus modos particulares de enfocar los problemas de investigación.

Si bien es verdad que hay un marco legislativo común que posibilita la homogeneidad en la oferta, el desarrollo y el reconocimiento final de los estudios superiores de tercer ciclo o doctorado universitario (ahora Máster de investigación), lo cierto es que un Programa de Doctorado Interuniversitario tiene sus dificultades administrativas porque han de ponerse de acuerdo tantos convenios, calendarios y modos de proceder como universidades se impliquen, en este caso, nueve. La organización del Programa que en estos momentos está extinto se corresponde con la normativa del Real Decreto 778/98 bajo la cual los estudios de tercer ciclo universitario se componían de dos cursos. Uno con veinte créditos de materias de carácter obligatorio y optativo, y un segundo curso en el que se debía realizar un "Trabajo de Investigación Tutelado" (TIT)

${ }^{7}$ El análisis realizado por Benayas, Gutiérrez y Hernández (2003: 71) sobre los nuevos planes de estudios publicados en el Boletín Oficial del Estado ha permitido identificar a las universidades que están impartiendo alguna materia relacionada con la Educación Ambiental: Islas Baleares (UIB), La Laguna (ULL), Complutense (UCM) y Autónoma de Madrid (UAM), Alcalá de Henares (UAH), Barcelona (UB) y Autónoma de Barcelona (UAB), Granada (UGR), Girona (UdG), Santiago de Compostela (USC), País Vasco (UPV), Salamanca (USA), León (ULE), Málaga (UMA), Almería (UAL), Sevilla (US), Oviedo (UNIOVI), Alicante (UA) y Valencia (UVA), a las que habría que añadir A Coruña (UDC) y Vigo (UVIGO) de Galicia. Las que figuran con sus siglas identificativas en negrita son las nueve que participan en el Programa de doctorado. 
equivalente a doce créditos. La defensa pública de este trabajo de investigación ante un tribunal de tres miembros del profesorado del Programa de doctorado suponía la finalización de los cursos y la obtención del Diploma de Estudios Avanzados (DEA).

La singularidad de este Programa Interuniversitario se acentuó con el lugar escogido para la celebración de la docencia presencial: el Centro Nacional de Educación Ambiental situado en una pequeña localidad denominada Valsaín en las cercanías de la ciudad histórica de Segovia. El Ministerio de Medio Ambiente a través de su Organismo Autónomo de Parques Naturales, facilitó el uso y financió la estancia de alumnado y profesorado en las instalaciones del CENEAM.

Para mejorar la organización en dos anualidades (una para formarse en los diferentes seminarios y otra para iniciarse en las tareas de investigación), se optó por concentrar en dos períodos presenciales las materias ofertadas. De este modo, las asignaturas obligatorias se cursaban en febrero y las optativas (combinando una oferta simultánea de materias de dos en dos) en el mes de julio. Esta secuencia favorecía la presencia del profesorado, dado que en sus universidades respectivas febrero y julio eran períodos de exámenes y no de docencia. Además, el alumnado disponía de tiempo previo para preparar las lecturas necesarias para el desarrollo de las materias y las búsquedas y trabajos posteriores a las mismas en los períodos no presenciales. La calidad e intensidad de la convivencia en las instalaciones del CENEAM no impedía al alumnado estar plenamente integrado como miembros de las universidades en las que formalizaban sus respectivas matrículas.

El cuadro que figura a continuación contiene información sobre las materias obligatorias y optativas del Programa de doctorado Interuniversitario de Educación Ambiental:

\begin{tabular}{|c|c|c|}
\hline $\begin{array}{c}\text { MATERIAS } \\
\text { OBLIGATORIAS }\end{array}$ & PROFESORADO & ÁREA DE CONOCIMIENTO \\
\hline $\begin{array}{c}\text { Modelos en Educación } \\
\text { Ambiental }\end{array}$ & $\begin{array}{l}\text { Pujol, Rosa M } \text { M }^{\mathrm{a}} \text { (UAB) } \\
\text { Geli, Ana María (UdG) } \\
\text { Junjent, Mercé (UAB) }\end{array}$ & $\begin{array}{c}\text { Didáctica de las Ciencias } \\
\text { Experimentales }\end{array}$ \\
\hline $\begin{array}{l}\text { Introducción a la } \\
\text { investigación en la } \\
\text { Educación Ambiental }\end{array}$ & $\begin{array}{l}\text { Gutiérrez, José (UGR) } \\
\text { Benayas, Javier (UAM) }\end{array}$ & $\begin{array}{c}\text { Métodos de Investigación en } \\
\text { Educación } \\
\text { Ecología }\end{array}$ \\
\hline $\begin{array}{c}\text { Fuentes de } \\
\text { información y } \\
\text { documentación en }\end{array}$ & $\begin{array}{c}\text { Sureda Negre, Jaume } \\
\text { (UIB) }\end{array}$ & $\begin{array}{c}\text { Didáctica y Organización } \\
\text { Escolar }\end{array}$ \\
\hline
\end{tabular}


educación ambiental

MATERIAS OPTATIVAS

PROFESORADO ÁREA DE CONOCIMIENTO

\begin{tabular}{|c|c|c|}
\hline $\begin{array}{c}\text { Modelos de } \\
\text { aprendizaje y } \\
\text { estrategias de } \\
\text { educación ambiental }\end{array}$ & $\begin{array}{l}\text { García, José Eduardo } \\
\text { (US) } \\
\text { Porlán, Rafael (US) } \\
\text { Rivero, Ana (US) } \\
\text { García, Francisco F. } \\
\text { (US) }\end{array}$ & $\begin{array}{c}\text { Didáctica de las Ciencias } \\
\text { Experimentales } \\
\text { Didáctica de las Ciencias Sociales }\end{array}$ \\
\hline & Calvo, Aina (UIB) & Didáctica y Organización Escolar \\
\hline $\begin{array}{c}\text { Educación para la } \\
\text { sostenibilidad y } \\
\text { desarrollo comunitario }\end{array}$ & $\begin{array}{c}\text { Meira Cartea, Pablo A. } \\
\text { (USC) } \\
\text { Vargas Callejas, Germán } \\
\text { (USC) } \\
\text { Caride Gómez, José } \\
\text { Antonio (USC) }\end{array}$ & Teoría e Historia de la Educación \\
\hline $\begin{array}{c}\text { Procesos de } \\
\text { percepción, } \\
\text { comprensión y } \\
\text { comunicación en el } \\
\text { campo de lo ambiental }\end{array}$ & $\begin{array}{l}\text { Pou Royo, Antonio } \\
\text { (UAM) }\end{array}$ & Ecología \\
\hline
\end{tabular}

Comportamiento y medio ambiente.

Conceptos y técnicas de investigación

Corraliza, José Antonio
(UAM)
Psicología Social

Berenguer Santiago, Jaime (UAM)

Educación, sociedad, tecnología y medio ambiente

Barroso Jerez, Clara

(ULL)
Teoría e Historia de la Educación
Educación Ambiental y sostenibilidad local
Iglesias da Cunha, Lucía (USC)

Hernández del Águila, Rafael (UGR)
Teoría e Historia de la Educación

Geografía física

\footnotetext{
Análisis y elaboración de programas $y$ materiales de divulgación científica y
}

Escalas, $\mathrm{M}^{\mathrm{a}}$ Teresa

(UAB)
Didáctica de las Ciencias

Experimentales 
Educación para el desarrollo sostenible desde los centros educativos
Aznar Minguet, Pilar (UV)

García Gómez, Javier (UV)
Teoría e Historia de la Educación

Didáctica de las Ciencias Experimentales

El conjunto responde a una oferta plausible y permite concretar los contenidos del programa de doctorado que se impartía. El enfoque de las materias a la hora de presentar los temas específicos que abordaban, era la investigación en el campo: metodologías, análisis de resultados, críticas a trabajos realizados, etc. Para actualizar este programa al nuevo formato de Máster en investigación sería necesario actualizar ciertos contenidos e introducir otros módulos/seminarios que pudiesen contribuir desde otros campos científicos como la sociología, la economía, el derecho y otras ciencias, para así capacitar desde una formación más completa sobre la problemática ambiental, los procesos educativos que se están desarrollando y las formas de investigarlos.

La oferta que realizaba el Programa Interuniversitario de Educación Ambiental era adecuada a las competencias asociadas a las titulaciones de Máster (formación en un campo disciplinar, aplicación del conocimiento en la resolución de problemas, comunicación y elaboración de juicios críticos sobre el área de conocimiento) (Barroso, 2008: 49). La transformación no ha sido posible por problemas de gestión administrativa y de autonomía en la política educativa universitaria de cada una de las Comunidades que componen el Estado español. La crisis financiera ha venido a agravar la situación porque no se permite detraer recursos económicos para sufragar ciertos gastos de desplazamiento del profesorado, ni se permite "computar" la docencia en los Máster de investigación en el plan docente anual del profesorado. Esto produce una cierta sobrecarga que redunda en la falta de tiempo material para cumplir con la elaboración de la documentación administrativa necesaria para presentar una propuesta en cada una de las universidades interesadas en seguir adelante con esta aventura formativa.

\section{TRABAJOS DE INVESTIGACIÓN Y TESIS DOCTORALES VINCULADAS AL PROGRAMA}

El perfil de los estudiantes de este Programa de doctorado concentra a un mayor número de profesionales en activo (80\%) que de estudiantes recién licenciados (17\%). Educadores ambientales de Centros y programas de Educación Ambiental (31\%), Técnicos de Medio Ambiente (20\%), profesorado de educación secundaria (14\%), profesorado de universidad $(13 \%)$, personal de la administración $(2,5 \%)$ y personas sin actividad laboral $(2,5 \%)$. Esta circunstancia 
podría ser favorable a la realización de investigaciones sobre la práctica, pero no siempre se dan las condiciones para poder combinar el trabajo profesional con la tarea de investigar. Además, la falta de ayudas o contratos de investigación hace desistir de la labor investigadora a favor de otras oportunidades laborales. En cierto modo, la actividad profesional del estudiantado de este programa inclina sus intereses hacia el perfeccionamiento de su formación más que hacia el inicio de una carrera profesional como investigadoras o investigadores (Barroso, 2008). Pero de modo idéntico sucede con muchos otros cursos de doctorado o de Máster de investigación en los que una parte del alumnado no tiene definida su identidad futura en el campo de la investigación.

Para el análisis de las características, contenidos y planteamientos específicos de los trabajos de investigación en Educación Ambiental podemos sintetizar las aportaciones de Javier Benayas (1996; 1997), José Gutiérrez (1997), Antonella Bachiorri (1996) y Lucie Sauvé (2000). Las claves de análisis serán los objetivos de investigación, los tópicos o temas más frecuentes, la tipología o el planteamiento general del trabajo y las técnicas específicas empleadas para recoger los datos que se manejan en las investigaciones realizadas (Iglesias, 2008: $57-58)$.

- Los objetivos de investigación se centran mayormente en el análisis o valoración de diferentes tipologías de programas de Educación Ambiental. Esta finalidad descriptiva se complementa con la de otros trabajos que pretenden definir un marco teórico-conceptual para la Educación Ambiental, y el de los que evalúan alguna de las dimensiones que afectan a las personas que participan en actividades de Educación Ambiental (cambios que experimentan los conocimientos, concepciones o ideas previas de los alumnos; o transformaciones en la escala de valores o en sus actitudes e intereses hacia temas ambientales). Son menos frecuentes los trabajos cuyos objetivos están relacionados con el estudio de los niveles de formación de la educadora o del educador ambiental, de sus propias actitudes y creencias con respecto a la Educación Ambiental, o de los niveles de participación ciudadana en temas relacionados con el medio ambiente. Cabe decir también que muchos de los trabajos de investigación que se realizan se plantean elaborar propuestas de actuación referidas al contexto institucional o social investigado (Iglesias, 2008: 57).

- Los tópicos o temas en los que la investigación en Educación Ambiental centra su interés suelen ser: aspectos relativos a la enseñanza y al aprendizaje dentro de la situación pedagógica que se genera en el aula o en el centro de Educación Ambiental; aspectos relacionados con la subjetividad del alumnado (actitudes, valores, representaciones y dominios conceptuales más que comportamentales), cuestiones referidas a la formación de las personas responsables de los procesos formativos (las formadoras y los formadores, valga 
la redundancia) y, finalmente, temas relacionados con los debates científicos sobre la gestión de los recursos naturales, la ética ambiental, la educación global, la población, el desarrollo sostenible, etc. Es claramente observable que los temas que han sido objeto de investigación en Educación Ambiental son plurales, no se definen bajo líneas uniformes sino que atienden a dimensiones múltiples y a acontecimientos casi siempre contingentes o derivados de intereses personales (Meira, 2000). Estos elementos circunstanciales ponen de manifiesto que no hay una agenda temática consensuada entre la comunidad de personas interesadas en la investigación del campo.

- La tipología de las investigaciones realizadas siguen un planteamiento general descriptivo en su mayor parte. También hay diseños experimentales y trabajos de carácter histórico-documental, así como estudios que se diseñan desde los presupuestos de la investigación-acción. Los modelos de investigación parecen debatirse entre lo cuantitativo y lo cualitativo. Los paradigmas que sirven de base a uno y otro parten de supuestos filosóficos diferentes acerca de la naturaleza y las posibilidades de interpretar la realidad. En general, puede decirse que existen varias tradiciones científicas: una positivista, otra de tipo interpretativo y una tercera denominada "crítica" que hace que los investigadores trabajen muchas veces de modo opuesto. Estos tipos de investigación son considerados muchas veces excluyentes entre sí, pero también hay invitaciones a su uso complementario a condición de que se reconozcan sus respectivos fundamentos y las implicaciones de éstos (Sauvé, 2000: 56).

- Las técnicas de investigación utilizadas siguen optando mayoritariamente por los cuestionarios y encuestas que suelen construirse tras una revisión bibliográfica sobre el tema que abordan. También existen un nutrido grupo de trabajos que sólo utilizan las técnicas bibliográficas y documentales como base informativa. En menor medida aparecen estudios que utilizan técnicas de carácter descriptivo-cualitativo, como por ejemplo, etnografías, observaciones pautadas o registros en vídeo. La presencia minoritaria de entrevistas personales o aplicación de pruebas proyectivas completan el cuadro de la diversidad en cuanto a las técnicas de investigación.

\section{DIVULGACIÓN CIENTÍFICA Y PUBLICACIONES DEL PROGRAMA INTERUNIVERSITARIO DE DOCTORADO EN EDUCACIÓN AMBIENTAL}

La estructura general de la producción científica se mide con parámetros de visibilidad documental y difusión. Las tres fuentes de información más importantes al respecto son: las comunicaciones a Congresos y Jornadas específicas del campo, las Tesis doctorales defendidas y los artículos publicados en revistas internacionales. En este sentido, la ausencia de revistas científicas que 
aborden la temática de la Educación Ambiental en lengua española ha limitado, en parte, la difusión de trabajos de investigación en este campo. La revista Tópicos en Educación Ambiental -de ámbito latinoamericano-, la de Ciclos -de ámbito español- y la de Ambientalmente sustentable -de ámbito galaicoportugués- surgieron progresivamente con el propósito de llenar este vacío (Iglesias, 2008: 55).

Desde el año 2005 al 2008 el Grupo de Investigación SEPA (Grupo de Investigación en Pedagogía Social y Educación Ambiental) de la Universidad de Santiago de Compostela organizó anualmente un encuentro de investigación en Educación Ambiental. La convocatoria del mismo era abierta para que toda la comunidad de personas que investigan en el campo de la Educación Ambiental se animasen a participar, pero particularmente las personas ligadas al Programa Interuniversitario de Educación Ambiental. La finalidad principal del evento era facilitar el encuentro, la exposición y la discusión entre pares de las Tesis de doctorado que se estaban desarrollando o de algunos Trabajos de Investigación finalizados por los ya investigadores e investigadoras noveles (Meira, 2001:13). De este modo se lograba un segundo objetivo que era divulgar y visibilizar el trabajo de investigación que las universidades participantes en el programa estaban desarrollando.

Por otro lado, el Programa cuenta con el apoyo del Ministerio de Medio Ambiente y del CENEAM para publicar una colección específica de libros donde figura prácticamente la totalidad de los Trabajos de Investigación Tutelados defendidos anualmente ante sus correspondientes tribunales. Esta defensa pública permite acceder, tal y como ya fue indicado antes, al reconocimiento del Diploma de Estudios Avanzados que posibilita la inscripción de una Tesis doctoral. En estos momentos hay publicados seis volúmenes en los que se han registrado 113 trabajos originales de investigación (de los 134 DEAs defendidos, lo que supone un $84 \%$ del volumen de investigación realizado). Es por ello que Pablo Meira (2009: 9) afirmaba en la presentación de uno de estos volúmenes que constituyen uno de los mayores esfuerzos colectivos de construcción del conocimiento en el campo de la Educación Ambiental realizados en el espacio cultural y lingüístico iberoamericano ${ }^{8}$.

${ }^{8}$ La lengua vehicular para la publicación de todos los trabajos en esta colección del CENEAM es el español, sin embargo, como las Universidades permiten el uso de las lenguas cooficiales (el catalán, el valenciano y el gallego) y, en el caso de la Universidad de Santiago de Compostela, el portugués (por su afinidad y proximidad a la lengua gallega), los trabajos originales se han presentado en diferentes lenguas. 
Es muy importante el contacto permanente entre equipos de investigación, de modo que se evite el aislamiento y amplifiquen la difusión del trabajo realizado. Por ello, los seminarios de investigación son necesarios y enriquecedores en el ámbito académico y en el personal. Ahora bien, hay que trazar estrategias que permitan difundir la investigación que se realiza más allá de las fronteras estatales. Para ello debemos estimar más y mejor los trabajos que se realizan, considerarlos de calidad y de interés para otras personas de este campo de investigación.

\section{BIBLIOGRAFÍA}

Bachiorri, Antonella (1996): Investigation in Environmental Education: Problems and Perspectives. En Congreso Internacional Estratexias e Prácticas en Educación Ambiental. Ponencias. Santiago de Compostela: Universidad de Santiago. Bradford University (UK). Tórculo Edicións. 279-291.

Barroso Jerez, Clara (2008): La investigación universitaria en educación ambiental y para la sostenibilidad: situación actual y perspectivas en el nuevo marco europeo y español de los estudios de Tercer Ciclo. En Meira, Pablo A y Andrade, Marilia, Investigación e Formación en Educación Ambiental. Novos escenarios e enfoques para un tempo de cambios. Santa Cruz-Oleiros: Centro de Extensión Universitaria e Divulgación Ambiental de Galicia (CEIDA). 43-50.

Benayas, Javier (1996): Análisis de las Tesis Doctorales sobre Educación Ambiental leídas en España. En Congreso Internacional Estratexias e Prácticas en Educación Ambiental. Ponencias. Santiago de Compostela: Universidad de Santiago. Bradford University (UK). Tórculo Edicións. 293-308.

Benayas, Javier (1997): Investigación y educación ambiental. En J. Gutiérrez et al., Líneas de investigación en Educación Ambiental. Granada: Universidad de Granada / Junta de Andalucía. Delegación Provincial de Medio Ambiente. 39-49.

Benayas, Javier; Gutiérrez, José; Hernández, Norma (2003): La investigación en educación ambiental en España. Madrid: Ministerio de Medio Ambiente.

Iglesias da Cunha, Lucía (2008): La investigación en Educación Ambiental: necesidad de un plan para un futuro de calidad. En Meira, Pablo A y Andrade, Marilia, Investigación e Formación en Educación Ambiental. Novos escenarios e enfoques para un tempo de cambios. Santa Cruz-Oleiros: Centro de Extensión Universitaria e Divulgación Ambiental de Galicia (CEIDA). 51-62.

Meira Cartea, Pablo (2000): La investigación en Educacón Ambientl y las Nuevas Tecnologías de la información y la Comunicación. Teoría de la Educación y cultura en la sociedad de la información, 2. Revista electrónica de la Universidad de Salamanca. http://www.usal.es/ teoriaeducacion/ 
Meira Cartea, Pablo (2008): Investigar en Educación Ambiental: dos proxectos ás redes. En Meira, Pablo y Andrade, Marilia, Investigación e Formación en Educación Ambiental. Novos escenarios e enfoques para un tempo de cambios. Santa Cruz-Oleiros: Centro de Extensión Universitaria e Divulgación Ambiental de Galicia (CEIDA). 9-16.

Meira Cartea, Pablo (Coord.) (2009). Educación Ambiental: Investigando sobre la práctica. Madrid: CENEAM. Ministerio de Medio Ambiente.

Ministerio de Educación. Secretaría General de Universidades (2010): Datos y Cifras del Sistema Universitario Español. Curso 2010-2011). Madrid: Ministerio de Educación. http://educacion.gob.es

Sauvé, Lucie (2000): Para construir un patrimonio de investigación en Educación Ambiental. Tópicos en Educación Ambiental. 2 (5). 51-69.

Artigo recebido em 12/09/2012

Artigo aprovado em 10/12/2012 